\title{
Comparison of Robot Versus Fluoroscopy-Assisted Pedicle Screw Instrumentation in Adolescent Idiopathic Scoliosis Surgery
}

\section{Canglong Hou}

Second Military Medical University First Hospital

\section{Yu Chen}

Huashan Hospital Fudan University

\section{Mingyuan Yang}

Second Military Medical University First Hospital

\section{Yilin Yang}

Second Military Medical University First Hospital

\section{Huan Yang}

Naval Medical University

\section{Kai Chen}

Naval Medical University

\section{Beichen Zhang}

The First Rehabilitation Hospital of Shanghai

\section{Kai Chen}

Second Military Medical University First Hospital

Ming Li ( $D$ liming_spine@126.com )

Second Military Medical University First Hospital https://orcid.org/0000-0002-3606-831X

\section{Research Article}

Keywords: adolescent idiopathic scoliosis, robot, fluoroscopy, pedicle screws

Posted Date: December 8th, 2021

DOI: https://doi.org/10.21203/rs.3.rs-1004618/v1

License: (a) (i) This work is licensed under a Creative Commons Attribution 4.0 International License. Read Full License 


\section{Abstract}

The aim of this study is to explore whether robot-assisted technique has advantages over conventional fluoroscopy-assisted technique in clinical and radiological outcomes and whether it could decrease the incidence of mis-implantations of pedicle screws in AlS correction surgery.

A total of 101 AlS patients were recruited (RA group: 45 patients underwent robot-assisted screw insertion; FA group: 56 patients underwent fluoroscopy-assisted screw insertion). When compared the radiological data between two groups, major and secondary curve were both corrected proficiently with no difference in the Cobb angle comparison at last follow-up, suggesting that both robot-assisted technique and fluoroscopy-assisted technique could lead to efficient radiographic correction and similar clinical outcomes (all, $P<0.05$ ). In RA group, operation time, blood loss and transfusion volume were significantly greater than those in FA group, while the accuracy of screw implantations in AIS patients with thoracic scoliotic curve in RA group was higher than that in FA group.

In conclusion, both robot-assisted and fluoroscopy-assisted technique could reach proficient radiographic correction and similar clinical outcomes in AIS surgery. Compared with conventional fluoroscopy technique, robot-assisted technique might improve the accuracy of screw implantations in AIS patients with thoracic scoliotic curve, while the increased operation time, blood loss and transfusion volume might be the disadvantages due to preliminary stage of learning curve.

\section{Introduction}

Adolescent idiopathic scoliosis (AIS) is the most common spinal deformity with the diverse prevalence ranging from $0.5-5.2 \%$ in pediatric population[1-3]. In the past decades, pedicle screw has been the predominant instrumentation in the AIS correction surgery. However, the screw misplacement has been constantly reported, with the incidence of $20 \%-30 \%$, even $1 \%$ of which even resulted in severe neurovascular complications[4-6]. Although robust preventative techniques, such as intraoperative x-ray[7], computed tomography[8] and electrophysiology monitor[9], have been employed in the AIS surgery, the screw insertion-related complications still give rise to great challenges on safety and satisfaction of AIS correction, such as injury of vessels and nerves. Therefore, how to decrease the incidence of misimplantation of pedicle screws and prevent the severe neurovascular complications remain challenges for spine surgeons.

In recent years, robot-assisted technology has been widely adopted in medical fields, bringing dramatic elevation of outcomes in surgery, which seems to provide perfect choice to reduce screw insertion-related complications and to obtain ideal correction effect with free radiation exposure for surgeon[10-12]. Croissant $Y$ et al.[13] used robot-assisted system for image-guided percutaneous $\mathrm{K}$-wire insertion in minimally invasive interventions of the spine, and their results suggested that robot assistance device performed high accuracy and safety during instrumentation, without any perforation of the pedicle wall. A Study by Shillingford et al.[14] demonstrated robotic-assisted S2Al screw placement as safe, accurate, and reliable for achieving solid spinopelvic fixation, and there was no difference in the total screw implant 
accuracy between free hand and robot-assisted technique (94.9\% versus $97.8 \%, P=0.630)$. A great deal of data has reported on the effectiveness and advancement of the robot-assisted technique in thoracic and lumbar spine surgery[12,15-19], while studies related to correction of scoliotic curves usually accompanied with dysplasia vertebrae pedicle are scarce. Therefore, it is essential to explore the accuracy of pedicle implantation of robot-assisted technique in AIS correction surgery to avoid the disadvantages of pedicle mal-implantations such as injury of vessels and nerves.

The present study aims to explore whether robot-assisted technique has advantages over conventional fluoroscopy-assisted technique in clinical and radiological outcomes and whether it could decrease the incidence of mis-implantation of pedicle screws in AIS correction surgery. We hope that our results could provide theories of effectiveness of robot-assisted technique in inserting accurate and secure pedicle screws in AlS surgery.

\section{Material And Methods}

\section{Patient recruitment}

The study was approved by the ethics committee of our university (Local Ethics Committee of Changhai Hospital, SMMU, No. CHEC2017-163). In accordance with the 1964 Helsinki declaration, the informed consents have been obtained from all participants and their parents or legal guardians when under 18 years old. The inclusion criteria were as follows: 1) AIS patients aged from 10 to 18; 2) patients with main curve Cobb angle more than 40 degree which needed posterior correction surgery; 3 ) with complete medical records of anteroposterior and lateral full spine X-ray, preoperative and postoperative CT scans. Other scoliosis types, such as neuromuscular scoliosis, syndrome scoliosis, were excluded from our study.

Before surgery, whether AIS patients would receive Renaissance robot system or traditional fluoroscopy was determined randomly by surgeons. Subjects were divided into 2 groups according to whether surgery was conducted with Renaissance robot system. Forty-five patients underwent robot-assisted (Renaissance ${ }^{\circledR}$, Mazor Robotics Ltd.; Caesarea, Israel) correction surgery (group RA), while the other fiftysix patients underwent pedicle screw insertion utilizing the free hand technique assisted by traditional fluoroscopy (group FA). In order to further investigate the effect of robot-assisted technology on operation, patients were further divided into subgroups on the basis of structural curve distribution: thoracic scoliosis (group RA 25, group FA 27), thoracolumbar/lumbar scoliosis (group RA 20, group FA 29). Considering minimum sample size estimation, we finally performed 46 cases of RA group, and 56 cases of FA group as control.

All surgery producers were performed by one experienced surgery team in single medical center. The whole subject population had at least one-year consecutive follow-up history.

The research methodology was shown in Figure 1.

\section{Fluoroscopy-Assisted technique}


The patients were placed prone on radiolucent surgery table and performed posterior midline incision to expose posterior elements at instrumented segments. According to principles proposed by Kim[20], the osseous anatomy was identified as a reference to choose screw entrance points. Following outer cortex penetration by high-speed burr, pedicle probe was directed perpendicular to the plane of the superior articular facet, and adjusted medial and caudal inclination to detect the safe screw trajectory[21]. At the same time, intraoperative imaging was conducted by $\mathrm{C}$-arm to verify the exact vertebrae segment and position of the pedicle screws inserted in coronal and sagittal planes. And rod contouring and translation in situ bending was performed in subsequent correction procedures, as well as appropriate compression or distraction if necessary.

\section{Robot-Assisted technique}

The Renaissance robot system consists of a controllable robot device, a stabilized platform and a surgical planning station for preoperative plan and intraoperative device motion control. Prior to the operation, the thin cut $(1 \mathrm{~mm})$ CT scan data of the planned instrumentation segments was transferred into the software, and the inserted screw dimensions and position have been confirmed according to pedicle parameters. After mounting the stabilized platform to the spinous process, two intraoperative x-ray films were used to define each vertebrae segment location. The controllable robot device moves to planned screw trajectory position and direction (shown in figure 2), and the pedicle screws were inserted according to the settled trajectory as drilled pathway. And later procedures were same as which described in fluoroscopy-assisted technique part.

\section{Data collection}

Baseline information were recorded including age, gender, Risser sign and Lenke types. Radiographic and clinical outcomes data were recorded in two groups preoperatively, immediate postoperatively and at last follow-up, including major curve and secondary curve of Cobb angle (the angle between major curve and secondary curve), thoracic kyphosis (TK, the Cobb angle between T5 and T12), lumbar lordosis (LL, he Cobb angle between L1 and L5), coronal balance (CB, the horizontal distance between the center of the S1 vertebra and a vertical line drawn from the center of C7 and C7-CSVL) and SVA (sagittal vertical axis, the horizontal offset from the posterosuperior corner of $\mathrm{S} 1$ to the vertebral body of $\mathrm{C7}$ ). Perioperative parameters including operation time, blood loss and transfusion volume during operation and postoperative complications were compared between two groups.

Thin cut $(1 \mathrm{~mm})$ CT scan was conducted postoperatively for all patients to evaluate the accuracy of screw placement. The misplacement grading system introduced by Abul-Kasim[22] classified screw malposition into 5 categories: medial cortical perforation (MCP), lateral cortical perforation (LCP), anterior cortical perforation (ACP), endplate perforation (EPP), and foraminal perforation (FP). In each category, grade 1 was identified with screws with partial cortical perforation, while grade 2 and ACP, EPP as total cortical perforation.

Complications such as screw loosening, adding-on, proximal junctional kyphosis (PJK) and revision surgery were also studied and compared between two groups. Besides, SRS-22 questionnaire was adopted to 
evaluate the health-related quality of life (HRQOL) in our study.

\section{Statistical Analysis}

Statistical analyses were performed using SPSS 22.0 statistics software (IBM Corp., Armonk, New York, USA). Descriptive statistics were listed in the form of mean and standard deviation (SD), and categorical data were listed in numbers. Paired sample t tests were conducted to analyze the differences between preoperative and postoperative parameters within groups. Independent two-sample t test was used to compare the differences of variables between two groups. $c^{2}$ test was used to compare the differences of count data. All statistical assessments were 2 -sided, and $P<0.05$ was considered with significant difference.

\section{Results}

\section{Baseline information}

A total of 101 AIS patients (male/female: $31 / 70$ ) were recruited into our study with a mean age of $14.58 \pm 2.00$ years. Of which all the subjects, 45 patients (Male:13, Female:32) were classified into group RA, while the other 56 patients (Male:18, Female:38) received FA technique. No significant difference was observed in age, gender, Risser sign and Lenke types between two groups as shown in Table 1 (all $P>0.05$ ). In addition, SRS-22 scores were also similar between two groups (3.96 \pm 0.52 vs $3.93 \pm 0.31, P=0.127)$.

\section{Comparisons of preoperative radiological parameters}

The major Cobb angle, secondary Cobb angle, TK and LL in RA group and FA group was $48.79 \pm 7.03^{\circ}$, $27.83 \pm 7.17^{\circ}, 19.37 \pm 3.78^{\circ}, 39.02 \pm 2.44^{\circ}$ and $47.14 \pm 6.27^{\circ}, 26.95 \pm 5.67^{\circ}, 20.30 \pm 3.48^{\circ}$ and $39.65 \pm 2.50^{\circ}$ respectively, with no significant difference (all $P>0.05$ ). In addition, we also did not find any significant difference in CB and SVA, as shown in Table 1 (all $P>0.05$ ).

\section{Surgery-related Parameters}

With respect to surgery-related parameters, the fusion level $(10.41 \pm 2.53$ vs $9.60 \pm 2.84, P=0.094)$ and instrumented pedicle screw $(14.51 \pm 2.29$ vs $14.26 \pm 2.07, P=0.464)$ in group RA were comparable to those of group FA. However, the operation time $(210.12 \pm 1.78 \mathrm{~min} 179.07 \pm 16.60 \mathrm{~min}, P<0.001)$, blood loss $(1063.07 \pm 200.04 \mathrm{ml} v s 804.56 \pm 137.17 \mathrm{ml}, P<0.001)$ and transfusion volumes $(946.09 \pm 113.72 \mathrm{ml} v s$ $729.61 \pm 147.56 \mathrm{ml}, P<0.001)$ in RA group was greater than those in FA group, suggesting robot-assist technique requires more operation time and consequently gives rise to more blood loss and transfusion volume. In addition, the postoperative complications were also recorded in the 2 years' follow, and our results showed that the incidence of the screw loosening, adding-on, proximal junctional kyphosis and revision surgery were $4.44 \%, 0,6.66 \%$ and $2.22 \%$ in group RA, while $7.14 \%, 5.36 \%, 7.14 \%$ and 0 in group FA, while there was no neurological complication occurred in two groups. All the data were shown in Table 1.

\section{Radiological assessment of pedicle screw insertion}


Evaluation the accuracy of pedicle screw instrumentation was shown in table 2. In group FA, 24 of 647screws was classified into MCP, 32 were LCP, 4 were AC P, 3 were EP, 2 were FP; while in group FA, 34 of 771screws was classified into MCP, 51 were LCP, 7 were ACP, 7 were EP, 4 were FP. The total malposition rate of group RA and group FA was $10.05 \%$ and $13.36 \%$, respectively, and there was no difference between two groups. While stratified into two scoliosis types defined by structural curve distribution, group RA demonstrated significantly lower malposition rate in thoracic scoliosis patients when compared with group FA (89.70\% vs $84.4 \%, P=0.033)$, although there was no difference existed in thoracolumbar/lumbar scoliosis patient population ( $90.68 \%$ vs $89.58 \%, P=0.454)$.

\section{Comparisons of radiological parameters pre and at final follow-up}

When compared the radiological data between two groups, major and secondary curve was both corrected proficiently, however, there were no difference in the Cobb angle of major curve and secondary curve at last follow-up. Although sagittal alignment (including thoracic kyphosis and SVA) and coronal balance was significantly improved at last follow-up in group RA and group FA, the results showed no significant difference between two groups. In addition, lumbar lordosis changed slightly from $39.02 \pm 2.44^{\circ}$ to $38.74 \pm 7.05^{\circ}$ in group RA, and from $39.65 \pm 2.50^{\circ}$ to $35.95 \pm 8.52^{\circ}$ in group FA, with no statistically significant difference between two groups at last follow-up.

The HRQOL scores remained stable in both group RA and group FA, and manifested no significant difference. The details were described in table 3.

\section{Discussion}

Due to the powerful three column correction force, pedicel screw has been widely applied in AIS correction surgery in the past decades. However, relatively higher incidence of malposition of pedicle screws have been reported by many researchers, which might result in severe neurovascular complications. AIS, as a three-dimensional spinal deformity, its relative narrow pedicle and abnormality of vertebrae rotation might be attributed to the high incidence of malposition of pedicle screws, which may lead to the incidence of neurovascular complications. When compared with thoracolumbar/lumbar scoliosis, researchers found that malposition of pedicle screws occurred more frequently in patients with thoracic scoliotic curves. De Blas[23] claimed that the threshold magnitude of thoracic pedicle was much lower than that of lumbar pedicle. Pedicel screw insertion of curve has been regarded as a great challenge for spine surgeons, which companied by potential vascular and neurological damage risk, especially in sever thoracic deformity population. Therefore, it is urgent to explore techniques to decrease the mis-implantation of pedicle screws and prevent the occurrence of vascular and neurological damages.

Several pedicle screw insertion-assisted techniques, such as 3D protype model and navigation system, have been widely applied in spine surgery to reduce the incidence of screw malposition. Although 3D protype model could provide more comprehensive structure of the complex structure, the surgeons couldn't have access to gain instant information of pedicles to adjust the directions and depth of inserted screws during the operation[24]. In addition, it has been reported that the accuracy of screw implantations of the 
navigation system only reached to $85 \%$ [25]. More importantly, the limited improvement of these techniques may not far outweigh the complicated calibration procedures. Therefore, more effective and practical screw insertion-assisted techniques should be explored and applied in surgery to minimize the screw misimplantation in AIS correction surgery.

Robot-assistant surgery technique, emerging as the new manipulation in spine surgery, has been one of most robust methods to improve screw insertion accuracy, decrease potential neurological complication risk and intraoperative radiation exposure since introduction into clinical practice[13,26, 27]. In Khan A et al.'s study[28], patients were divided into two distinct groups to compared robotic technology with 3D computed tomography navigation in degenerative disk diseases. One group consisted of 50 patients underwent pedicle screw insertion guided by robot, and 189 screws of all 190 inserted pedicle screws were classified into Ravi I grade, and 1 screw was grade II. While in the other group consisted of 49 patients underwent surgery with the assistance of 3D-CT navigation system, there were 157 of all 165 screws was classified into Ravi grade I, and 8 screws grade II. The results suggested there was no significant difference in field of screw insertion accuracy $(P=0.11)$ between groups, whereas robot techniques could decrease the radiation dose, time of per-screw insertion and length of hospital stay than 3D-CT navigation. In addition, many studies have demonstrated the superiority of robot-assisted technique to conventional manipulations in general spine surgeries. As far as we know, no study has been conducted to compare the clinical and radiological outcomes between robot-assisted technique and conventional methods in AIS correction surgery which might be huge challenges for spine surgeons. The aim of this study is to explore the effectiveness of robot system in AIS surgery, compare the accuracy of screw insertion between robot and conventional fluoroscopy and the radiological and clinical parameters were also explored as well.

In our study, there was no difference in field of baseline information, preoperative radiographic parameters, fusion level and number of instrumented screws (all, $P<0.001$ ) between group RA and group FA, suggesting that the study populations of two groups came from the same AIS cohorts and underwent correction surgery with same strategy. Therefore, the selection bias and other biases which might impacts our results like operation factors were controlled. However, the operation time, blood loss and transfusion volumes in group RA were significantly greater than those of group FA, which was consistent with the result of Le et al.'s[29], Ghasem et al.'s[30] and Fan et al.'s study[31]. This finding might result from the ralatively complicated procedures of Robots compared with group FA., such as the installation of working panel, match with pre-operative CT films and et al. However, Hyun SJ et al.[32] reported a 1.5 min decreased in per screw insertion time between their first 15 robot-assisted cases and later 15 cases. It seemed that the prolonged operation time was decreased as total robot-assisted surgery cases rose, which might result from the effects of robot learning curve[30]. In our opinions, the operation time, blood loss and transfusion volumes might decrease in company with the increase of our cases and experience due to the learning curves.

With respect to postoperative complications, there were no significant difference was observed between two groups $(P=0.232)$. As to HRQOL, there was no significant difference in the SRS-22 scores at last follow up between two groups, manifesting the comparable clinical results in group RA and group FA in short follow-up durations. 
As to the accuracy of implantation of pedicle screws, the total screw malposition rate was $10.05 \%$ in group RA and $13.36 \%$ in group FA, nearly approaching significant difference between two groups $(P=0.054)$, which suggested that robot-assisted technique seemed hardly to claim significantly superior to conventional manipulations in radiological screw insertion accuracy, and the result was similar to studies of Hyun SJ et al's[32] and Park SM et al's[33]. The malposition rate in our study was lower than those of other studies on degenerative disk diseases, which could due to the dysplasia pedicle and vertebrae rotation in AIS patients[32, 34,35]. In addition, the recruited patients in studies might also influence the malposition rate of pedicle screws since each patient might have unique structure of pedicles. When we stratified these AIS patients into thoracic scoliosis and thoracolumbar/lumbar scoliosis, our results showed that robot-assisted technique could significantly decrease the malposition rate in AIS with thoracic scoliosis $(P=0.033)$, whereas no significant difference in thoracolumbar/lumbar scoliosis subgroup was observed $(P=0.454)$. The implantation of pedicel screws in thoracic curves is difficult than that in thoracolumbar/lumbar scoliosis due to the smaller pedicles of thoracic vertebrae and nearer locations by vessels and verves compared with that of thoracolumbar/lumbar vertebrae. Therefore, in RA group, implantations of pedicles were conducted by preoperative CT films, which could provide more accurate information about the pedicles. Besides, the higher malposition rate of thoracic scoliosis in group FA was attributed to more dysplastic vertebral morphometry existed in thoracic segment than lumbar scoliosis, which was supported by the results of de Blas et al's[23] ,Shaw KA et al's[26] and Abul-Kasim K et al's study[36].

The major curve, secondary curve, thoracic kyphosis, coronal balance and sagittal balance in AIS patients were significantly corrected after surgery in both groups (all, $P<0.001$ ), and there was no difference in these postoperative parameters between two groups. These findings suggested that both robot-assisted and fluoroscopy-assisted technique could reach proficient radiographic correction and similar clinical outcomes in AIS surgery. However, the lumbar lordosis in group RA $(P=0.818)$ and group FA $(P=0.257)$ only manifested slight change after correction, and suggested no significant difference at last follow-up in two groups $(P=0.286)$.

Although meaningful findings were observed and reported in our study, there were some potential limitations in our study should be addressed. Firstly, all 101 AIS patients were recruited from one single spine surgery center, which might not represent the whole conditions of AIS correction surgery assisted by robot or fluoroscopy system when considered the varied correction strategies and experiences on robot manipulation. In addition, lack of long- term follow-up data, as well as the relatively small patients sample size in our study, restricted comparison conducted for further radiological and clinical outcomes, therefore, persistent follow-up research with larger AIS population should be performed.

\section{Typical case}

A typical case was presented in figure 3 .

\section{Conclusion}


In conclusion, both robot-assisted and fluoroscopy-assisted technique could lead to proficient radiographic correction and similar clinical outcomes in AIS surgery. Compared with conventional fluoroscopy technique, Robot-assisted technique might improve the accuracy of screw implantations in AIS patients with thoracic scoliotic curve, while increasing operation time, blood loss and transfusion volume during operation due to preliminary stage of learning curve.

\section{Abbreviations}

AIS: adolescent idiopathic scoliosis

RA: robot-assisted

FA: free hand technique assisted by traditional fluoroscopy

TK: thoracic kyphosis

LL: lumbar lordosis

CB: coronal balance

SVA: sagittal vertical axis

MCP: medial cortical perforation

LCP: lateral cortical perforation

ACP: anterior cortical perforation

EPP: endplate perforation

FP: foraminal perforation

PJK: proximal junctional kyphosis

HRQOL: health-related quality of life

SD: standard deviation

\section{Declarations}

\section{Ethics approval and consent to participate}

The study was approved by the ethics committee of our university (Local Ethics Committee of Changhai Hospital, SMMU, No. CHEC2017-163).

\section{Consent for publication}


Not applicable.

\section{Availability of data and materials}

All data generated or analysed during this study are included in this. published article

\section{Competing interests}

The authors declare that they have no competing interests

\section{Funding}

Shanghai Sailing Program (19YF1447100) and National Natural Science Foundation of China (No.81501845) are acknowledged for providing funding.

\section{Authors' contributions}

Conception and study design: Kai Chen and Ming Li. Acquisition of data: Mingyuan Yang, Kai Chen, Beichen Zhang. Analysis of data: Yilin Yang,Huan Yang, Canglong Hou, Yu Chen. Drafting of the manuscript: Canglong Hou. Critical revision of manuscript and approval of final version to be published: All authors. Final approval of the version published: All authors. Kai Chen and Ming Li had full access to all the data in the study and takes full responsibility for the integrity and the accuracy of data analysis

\section{Acknowledgements}

Shanghai Sailing Program (19YF1447100) and National Natural Science Foundation of China (No.81501845) are acknowledged for providing funding. The funding sources had no involvement in the design, data collection, data analysis, and manuscript preparation and/or publication decisions.

\section{References}

1. Tarrant RC, Queally JM, Moore DP, Kiely PJ. Prevalence and impact of low body mass index on outcomes in patients with adolescent idiopathic scoliosis: a systematic review. European journal of clinical nutrition2018 Nov;72(11):1463-84. doi:10.1038/s41430-018-0095-0

2. Penha PJ, Ramos N, de Carvalho BKG, Andrade RM, Schmitt ACB, João SMA. Prevalence of Adolescent Idiopathic Scoliosis in the State of São Paulo, Brazil. Spine2018 Dec 15;43(24):1710-18. doi:10.1097/brs.0000000000002725

3. Komang-Agung IS, Dwi-Purnomo SB, Susilowati A. Prevalence Rate of Adolescent Idiopathic Scoliosis: Results of School-based Screening in Surabaya, Indonesia. Malaysian orthopaedic journal2017 Nov;11(3):17-22. doi:10.5704/moj.1711.011 
4. Winder MJ, Gilhooly PM. Accuracy of minimally invasive percutaneous thoracolumbar pedicle screws using 2D fluoroscopy: a retrospective review through 3D CT analysis. Journal of spine surgery (Hong Kong)2017 Jun;3(2):193-203. doi:10.21037/jss.2017.06.05

5. Pan Y, Lü GH, Kuang L, Wang B. Accuracy of thoracic pedicle screw placement in adolescent patients with severe spinal deformities: a retrospective study comparing drill guide template with free-hand technique. European spine journal : official publication of the European Spine Society, the European Spinal Deformity Society, and the European Section of the Cervical Spine Research Society2018 Feb;27(2):319-26. doi:10.1007/s00586-017-5410-2

6. Ishak B, Younsi A, Wieckhusen C, Slonczewski P, Unterberg AW, Kiening KL. Accuracy and revision rate of intraoperative computed tomography point-to-point navigation for lateral mass and pedicle screw placement: 11-year single-center experience in 1054 patients. Neurosurgical review2019 Dec;42(4):895-905. doi:10.1007/s10143-018-01067-z

7. Newell R, Esfandiari H, Anglin C, Bernard R, Street J, Hodgson AJ. An intraoperative fluoroscopic method to accurately measure the post-implantation position of pedicle screws. International journal of computer assisted radiology and surgery2018 Aug;13(8):1257-67. doi:10.1007/s11548-018-1732-8

8. Bauer JM, Moore JA, Rangarajan R, Gibbs BS, Yorgova PK, Neiss GI et al. Intraoperative CT Scan Verification of Pedicle Screw Placement in AIS to Prevent Malpositioned Screws: Safety Benefit and Cost. Spine deformity2018 Nov-Dec;6(6):662-68. doi:10.1016/j.jspd.2018.04.010

9. Iyori K, Kamiya K, Suzuki O, Kojima A, Kubota K, Ishimoto T et al. Identification of the critical spinal arteries with $\mathrm{F}$ wave-polysynaptic response complex monitoring: an experimental study. Journal of vascular surgery2001 Aug;34(2):330-6. doi:10.1067/mva.2001.114816

10. Vahdat S, Darainy M, Thiel A, Ostry DJ. A Single Session of Robot-Controlled Proprioceptive Training Modulates Functional Connectivity of Sensory Motor Networks and Improves Reaching Accuracy in Chronic Stroke. Neurorehabilitation and neural repair2019 Jan;33(1):70-81. doi:10.1177/1545968318818902

11. Han JH, Lee JH, Hwang DW, Song KB, Shin SH, Kwon JW et al. Robot resection of a choledochal cyst with Roux-en-y hepaticojejunostomy in adults: Initial experiences with 22 cases and a comparison with laparoscopic approaches. Annals of hepato-biliary-pancreatic surgery2018 Nov;22(4):359-66. doi:10.14701/ahbps.2018.22.4.359

12. Troude L, Boissonneau S, Malikov S, Champsaur P, Blondel B, Dufour H et al. Robot-assisted multi-level anterior lumbar interbody fusion: an anatomical study. Acta neurochirurgica2018 Oct;160(10):1891-98. doi:10.1007/s00701-018-3621-x

13. Croissant Y, Zangos S, Albrecht MH, Eichler K, Schomerus C, Spandorfer A et al. Robot-assisted percutaneous placement of K-wires during minimally invasive interventions of the spine. Minimally invasive therapy \& allied technologies : MITAT : official journal of the Society for Minimally Invasive Therapy 2019 Dec;28(6):373-80. doi:10.1080/13645706.2018.1544567 
14. Shillingford JN, Laratta JL, Park PJ, Lombardi JM, Tuchman A, Saifi C et al. Human versus Robot: A Propensity-Matched Analysis of the Accuracy of Free Hand versus Robotic Guidance for Placement of S2 Alar-lliac (S2AI) Screws. Spine2018 Nov 1;43(21):E1297-e304. doi:10.1097/brs.0000000000002694

15. Wu C, Lee CY, Huang TJ, Wu MH. Cement-augmented pedicle screw insertion assisted by spinal robotic systems for widespread spinal metastases. Journal of robotic surgery2019 Aug;13(4):595-98. doi:10.1007/s11701-018-0886-2

16. Van't Hullenaar CDP, Bos P, Broeders I. Ergonomic assessment of the first assistant during robotassisted surgery. Journal of robotic surgery2019 Apr;13(2):283-88. doi:10.1007/s11701-018-0851-0

17. Liu HS, Duan SJ, Liu SD, Jia FS, Zhu LM, Liu MC. Robot-assisted percutaneous screw placement combined with pelvic internal fixator for minimally invasive treatment of unstable pelvic ring fractures. The international journal of medical robotics + computer assisted surgery : MRCAS2018 Oct;14(5):e1927. doi:10.1002/rcs.1927

18. Khan A, Meyers JE, Siasios I, Pollina J. Next-Generation Robotic Spine Surgery: First Report on Feasibility, Safety, and Learning Curve. Operative neurosurgery (Hagerstown, Md)2019 Jul 1;17(1):61-69. doi:10.1093/ons/opy280

19. Ha Y. Robot-Assisted Spine Surgery: A Solution for Aging Spine Surgeons. Neurospine2018 Sep;15(3):187-88. doi:10.14245/ns.18edi.003

20. Kim YJ, Lenke LG, Cheh G, Riew KD. Evaluation of pedicle screw placement in the deformed spine using intraoperative plain radiographs: a comparison with computerized tomography. Spine2005 Sep 15;30(18):2084-8. doi:10.1097/01.brs.0000178818.92105.ec

21. Etemadifar M, Jamalaldini M. Evaluating Accuracy of Free-hand Pedicle Screw Insertion in Adolescent Idiopathic Scoliosis Using Postoperative Multi-Slice Computed Tomography Scan. Advanced biomedical research2017;6:19. doi:10.4103/2277-9175.201331

22. Abul-Kasim K, Strömbeck A, Ohlin A, Maly P, Sundgren PC. Reliability of low-radiation dose CT in the assessment of screw placement after posterior scoliosis surgery, evaluated with a new grading system. Spine2009 Apr 20;34(9):941-8. doi:10.1097/BRS.0b013e31819b22a4

23. de Blas G, Barrios C, Regidor I, Montes E, Burgos J, Pizá-Vallespir G et al. Safe pedicle screw placement in thoracic scoliotic curves using t-EMG: stimulation threshold variability at concavity and convexity in apex segments. Spine2012 Mar 15;37(6):E387-95. doi:10.1097/BRS.0b013e31823b077b

24. Yang M, Li C, Li Y, Zhao Y, Wei X, Zhang G et al. Application of 3D rapid prototyping technology in posterior corrective surgery for Lenke 1 adolescent idiopathic scoliosis patients. Medicine2015 Feb;94(8):e582. doi:10.1097/md.0000000000000582 
25. Elmi-Terander A, Skulason H, Söderman M, Racadio J, Homan R, Babic D et al. Surgical Navigation Technology Based on Augmented Reality and Integrated 3D Intraoperative Imaging: A Spine Cadaveric Feasibility and Accuracy Study. Spine2016 Nov 1;41(21):E1303-e11. doi:10.1097/brs.0000000000001830

26. Shaw KA, Murphy JS, Devito DP. Accuracy of robot-assisted pedicle screw insertion in adolescent idiopathic scoliosis: is triggered electromyographic pedicle screw stimulation necessary? Journal of spine surgery (Hong Kong)2018 Jun;4(2):187-94. doi:10.21037/jss.2018.04.01

27. Kim HJ, Jung WI, Chang BS, Lee CK, Kang KT, Yeom JS. A prospective, randomized, controlled trial of robot-assisted vs freehand pedicle screw fixation in spine surgery. The international journal of medical robotics + computer assisted surgery : MRCAS2017 Sep;13(3). doi:10.1002/rcs.1779

28. Khan A, Meyers JE, Yavorek S, O'Connor TE, Siasios I, Mullin JP et al. Comparing Next-Generation Robotic Technology with 3-Dimensional Computed Tomography Navigation Technology for the Insertion of Posterior Pedicle Screws. World neurosurgery2019 Mar;123:e474-e81. doi:10.1016/j.wneu.2018.11.190

29. Le X, Tian W, Shi Z, Han X, Liu Y, Liu B et al. Robot-Assisted Versus Fluoroscopy-Assisted Cortical Bone Trajectory Screw Instrumentation in Lumbar Spinal Surgery: A Matched-Cohort Comparison. World neurosurgery2018 Dec;120:e745-e51. doi:10.1016/j.wneu.2018.08.157

30. Ghasem A, Sharma A, Greif DN, Alam M, Maaieh MA. The Arrival of Robotics in Spine Surgery: A Review of the Literature. Spine2018 Dec 1;43(23):1670-77. doi:10.1097/brs.0000000000002695

31. Fan Y, Du J, Zhang J, Liu S, Xue X, Huang Y et al. Comparison of Accuracy of Pedicle Screw Insertion Among 4 Guided Technologies in Spine Surgery. Medical science monitor : international medical journal of experimental and clinical research2017 Dec 16;23:5960-68. doi:10.12659/msm.905713

32. Hyun SJ, Kim KJ, Jahng TA, Kim HJ. Minimally Invasive Robotic Versus Open Fluoroscopic-guided Spinal Instrumented Fusions: A Randomized Controlled Trial. Spine2017 Mar 15;42(6):353-58. doi:10.1097/brs.0000000000001778

33. Park SM, Kim HJ, Lee SY, Chang BS, Lee CK, Yeom JS. Radiographic and Clinical Outcomes of RobotAssisted Posterior Pedicle Screw Fixation: Two-Year Results from a Randomized Controlled Trial. Yonsei medical journal2018 May;59(3):438-44. doi:10.3349/ymj.2018.59.3.438

34. Fitzgerald R, Upasani VV, Bastrom TP, Bartley CE, Reighard FG, Yaszay B et al. Three-Dimensional Radiographic Analysis of Two Distinct Lenke 1A Curve Patterns. Spine deformity2019 Jan;7(1):66-70. doi:10.1016/j.jspd.2018.06.005

35. Chan A, Parent E, Lou E. Reconstruction and positional accuracy of 3D ultrasound on vertebral phantoms for adolescent idiopathic scoliosis spinal surgery. International journal of computer assisted radiology and surgery2019 Mar;14(3):427-39. doi:10.1007/s11548-018-1894-4 
36. Abul-Kasim K, Ohlin A, Strömbeck A, Maly P, Sundgren PC. Radiological and clinical outcome of screw placement in adolescent idiopathic scoliosis: evaluation with low-dose computed tomography. European spine journal : official publication of the European Spine Society, the European Spinal Deformity Society, and the European Section of the Cervical Spine Research Society2010 Jan;19(1):96-104.

doi:10.1007/s00586-009-1203-6

\section{Tables}

Table 1. The baseline information, preoperative radiological parameters, surgery-related parameters, complications and HRQOL in two groups 


\begin{tabular}{|llll|}
\hline Variables & Group RA (n=45) & Group FA (n=56) & Pvalue \\
\hline Baseline information & & & \\
\hline Age (years) & $14.69 \pm 1.93$ & $14.49 \pm 2.01$ & 0.587 \\
\hline Gender (Fmale/male) & $32 / 13$ & $38 / 18$ & 0.725 \\
\hline Risser ( $\left.{ }^{\circ}\right)$ & $1.34 \pm 0.90$ & $1.41 \pm 0.93$ & 0.710 \\
\hline Lenke types (1/2/3/4/5/6) & $22 / 8 / 4 / 1 / 7 / 3$ & $25 / 10 / 4 / 0 / 13 / 4$ & 0.823 \\
\hline Preoperative Radiological parameters & & & \\
\hline Cobb Angel (o) & & $47.14 \pm 6.27$ & 0.113 \\
\hline Major Curve & $48.79 \pm 7.03$ & $26.95 \pm 5.67$ & 0.512 \\
\hline Secondary Curve & $27.83 \pm 7.17$ & $20.30 \pm 3.48$ & 0.111 \\
\hline TK & $19.37 \pm 3.78$ & $39.65 \pm 2.50$ & 0.159 \\
\hline LL & $39.02 \pm 2.44$ & $11.36 \pm 0.31$ & 0.881 \\
\hline CB (mm) & $11.42 \pm 2.13$ & $20.44 \pm 3.21$ & 0.900 \\
\hline SVA (mm) & $20.37 \pm 3.42$ & & \\
\hline Surgery-related Parameters & & $3.93 \pm 0.31$ & 0.127 \\
\hline Fusion Level & $10.41 \pm 2.53$ & $9.60 \pm 2.84$ & 0.094 \\
\hline Instrumented Screws & $14.51 \pm 2.29$ & $14.26 \pm 2.07$ & 0.464 \\
\hline Operation Time (min) & $210.12 \pm 11.78$ & $179.07 \pm 16.60$ & $<0.001$ \\
\hline Blood Loss (ml) & $1063.07 \pm 200.04$ & $804.56 \pm 137.17$ & $<0.001$ \\
\hline Transfusion Volumes (ml) & $946.09 \pm 113.72$ & $729.61 \pm 147.56$ & $<0.001$ \\
\hline Complications & & & 0.232 \\
\hline Screw Loosening & $2(4.44 \%)$ & $4(7.14 \%)$ & \\
\hline Adding-on & $0(0)$ & $3(5.36 \%)$ & \\
\hline Proximal Junctional Kyphosis & $3(6.66 \%)$ & & \\
\hline Revision Surgery & $1(2.22 \%)$ & & \\
\hline HRQOL & & & \\
\hline SRS-22 Scores & & & \\
\hline
\end{tabular}

Group RA= robot-assisted screw insertion group; Group FA= fluoroscopy-assisted screw insertion group; $\mathrm{TK}=$ thoracic kyphosis; $\mathrm{LL}=$ lumbar lordosis; $\mathrm{CB}=$ coronal balance; $\mathrm{SVA}=$ sagittal vertical axis; $\mathrm{HRQOL}=$ health-related quality of life 
Table 2. Results of the radiological assessment of pedicle screw insertion between two groups

\begin{tabular}{|c|c|c|c|}
\hline & Group RA (n=45) & Group FA $(n=56)$ & Pvalue \\
\hline \multicolumn{4}{|l|}{ Total } \\
\hline Pedicle perforation & $56(8.66 \%)$ & $85(11.02 \%)$ & \\
\hline Medial cortical perforation (MCP) & $24(3.71 \%)$ & $34(4.41 \%)$ & \\
\hline Grade 1 & $16(2.47 \%)$ & $2(3.11 \%)$ & \\
\hline Grade 2 & $8(1.24 \%)$ & $10(1.29 \%)$ & \\
\hline Later Cortical perforation (LCP) & $32(4.95 \%)$ & $51(6.61 \%)$ & \\
\hline Grade 1 & $22(3.40 \%)$ & $33(4.28 \%)$ & \\
\hline Grade 2 & $10(1.55 \%)$ & $18(2.33 \%)$ & \\
\hline Anterior cortical perforation (ACP) & $4(0.62 \%)$ & $7(0.91 \%)$ & \\
\hline Endplate perforation (EP) & $3(0.46 \%)$ & $7(0.91 \%)$ & \\
\hline Foraminal perforation (FP) & $2(0.31 \%)$ & $4(0.52 \%)$ & \\
\hline Malposition screws & $65 \square 10.05 \% \square$ & $103(13.36 \%)$ & \\
\hline Image satisfactory screws & $584 \square 90.26 \% \square$ & $670(86.90 \%)$ & \\
\hline Sum up (all screws included in study) & $647 \square 100 \% \square$ & $771(100 \%)$ & 0.054 \\
\hline \multicolumn{4}{|l|}{ Thoracic scoliosis } \\
\hline Pedicle perforation & $33(8.94 \%)$ & $49(12.66 \%)$ & \\
\hline Medial cortical perforation (MCP) & $15(4.07 \%)$ & $19(4.91 \%)$ & \\
\hline Grade 1 & $12(3.25 \%)$ & $13(3.36 \%)$ & \\
\hline Grade 2 & $3(0.81 \%)$ & $6(1.55 \%)$ & \\
\hline Later Cortical perforation (LCP) & $18(4.88 \%)$ & $30(7.75 \%)$ & \\
\hline Grade 1 & $12(3.25 \%)$ & $19(4.91 \%)$ & \\
\hline Grade 2 & $6(1.63 \%)$ & $11(2.84 \%)$ & \\
\hline Anterior cortical perforation (ACP) & $3(0.81 \%)$ & $5(1.29 \%$ & \\
\hline Endplate perforation (EP) & $2(0.54 \%)$ & $4(1.03 \%)$ & \\
\hline Foraminal perforation (FP) & $1(0.27 \%)$ & $2(0.52 \%$ & \\
\hline Malposition screws & $38(10.30 \%)$ & $60(15.50 \%)$ & \\
\hline Image satisfactory screws & $331(89.70 \%)$ & $327(84.50 \%$ & \\
\hline Sum up (all screws included in study) & $369(100 \%)$ & $387(100 \%)$ & 0.033 \\
\hline \multicolumn{4}{|l|}{ Thoracolumbar/lumbar scoliosis } \\
\hline Pedicle perforation & $23(8.24 \%)$ & $36(9.38 \%)$ & \\
\hline Medial cortical perforation (MCP) & $9(2.23 \%)$ & $15(3.91 \%)$ & \\
\hline Grade 1 & $4(1.43 \%)$ & $11(2.86 \%)$ & \\
\hline Grade 2 & $5(1.79 \%)$ & $4(1.04 \%)$ & \\
\hline Later Cortical perforation (LCP) & $14(5.02 \%)$ & $21(5.47 \%)$ & \\
\hline Grade 1 & $10(3.58 \%)$ & $14(3.65 \%)$ & \\
\hline Grade 2 & $4(1.43 \%)$ & $7(1.82 \%)$ & \\
\hline Anterior cortical perforation (ACP) & $1(0.36 \%)$ & $2(0.52 \%)$ & \\
\hline Endplate perforation (EP) & $1(0.36 \%)$ & $3(0.78 \%)$ & \\
\hline Foraminal perforation (FP) & $1(0.36 \%)$ & $2(0.52 \%)$ & \\
\hline Malposition screws & $26(9.32 \%)$ & $43(11.20 \%)$ & \\
\hline Image satisfactory screws & $253(90.68 \%)$ & $344(89.58 \%)$ & \\
\hline Sum up (all screws included in study) & $279(100 \%)$ & $384(100 \%)$ & 0.454 \\
\hline
\end{tabular}

Group RA= robot-assisted screw insertion group; Group FA= fluoroscopy-assisted screw insertion group Table 3. Preoperative and last follow-up parameters between group RA and group FA

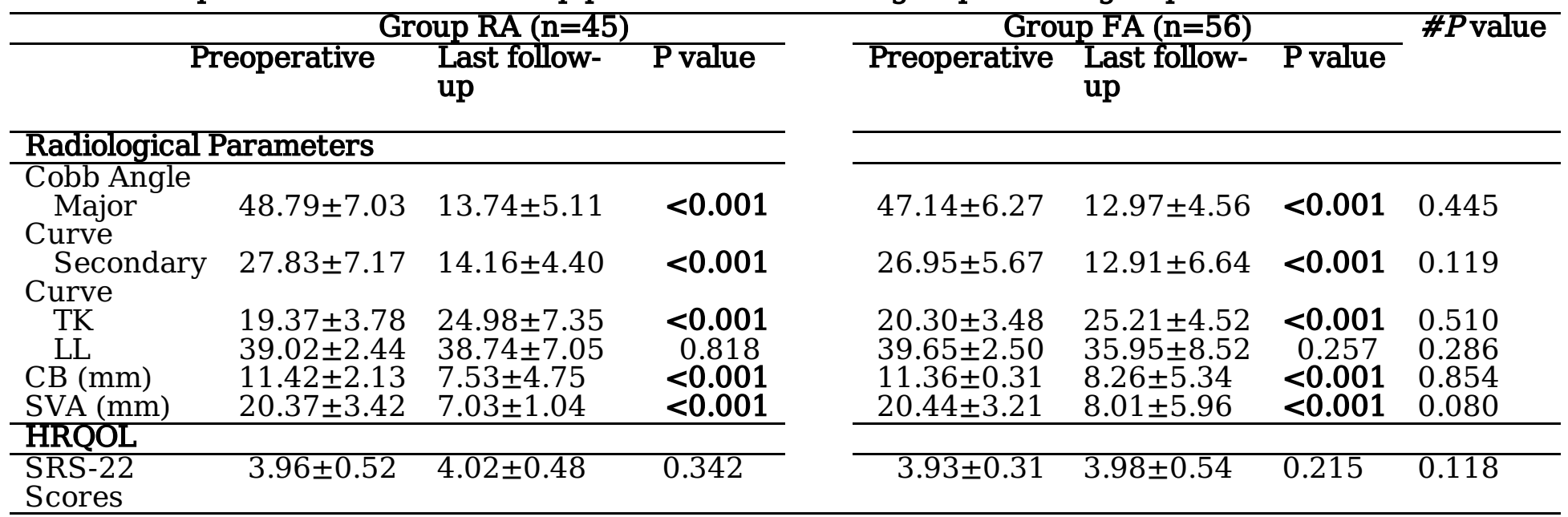

Group RA= robot-assisted screw insertion group; Group FA= fluoroscopy-assisted screw insertion group; $\mathrm{TK}=$ thoracic kyphosis; $\mathrm{LL}=$ lumbar lordosis; $\mathrm{CB}=$ coronal balance; SVA= sagittal vertical axis; HRQOL= 
health-related quality of life; \# P value= $\mathrm{p}$ value of last follow-up parameters between group RA and group FA.

\section{Figures}

\section{Figure 1}

Flowchart showed the research methodology

\section{Figure 2}

The controllable device of Renaissance robot system moves to planned screw trajectory position and direction on the basis of preoperative CT image, then pedicle screws were inserted according to the settled trajectory as drilled pathway. 

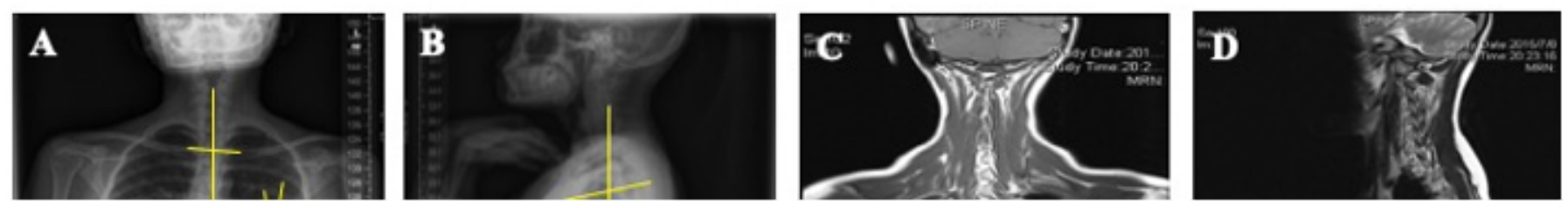

\section{Figure 3}

This picture showed the clinical pre-operative picture of this scoliosis patient. A 14-year old female Lenke 1 AIS patient underwent robot-assisted scoliosis correction surgery. Preoperative anteroposterior $(A)$ and lateral (B) full spine X-ray showed a Cobb angle of $42^{\circ}$ in major thoracic curve and $33^{\circ}$ in secondary lumbar curve (the angle between major curve and secondary curve). Coronal (C) and sagittal (D) view of full spine MRI showed there was no abnormalities within spinal cord, which supported the diagnose of AIS when combined with other clinical characteristics. Postoperative anteroposterior $(E)$ and lateral $(F)$ full spine Xray showed major thoracic curve was corrected to $10^{\circ}$, while secondary curve was corrected to $8^{\circ}$. Postoperative CT scan was used to evaluate the accuracy of screw insertion, and the typical image of satisfactory position $(G)$ and lateral cortical perforation $(H)$ were shown. In addition, clinical images of the patients both pre-operatively $(\mathrm{I})$ and post-operatively $(\mathrm{J})$ were also shown in Figure 3 . 